\title{
Cuts and Feynman amplitudes beyond polylogarithms
}

\author{
Johannes Broedel, ${ }^{a}$ Claude Duhr, ${ }^{b c}$ Falko Dulat, ${ }^{d}$ Brenda Penante ${ }^{b}$, Amedeo Primo ${ }^{e}$ \\ and Lorenzo Tancredith \\ ${ }^{a}$ Institut für Mathematik und Institut für Physik, Humboldt-Universität zu Berlin, \\ IRIS Adlershof, Zum Grossen Windkanal 6, 12489 Berlin, Germany \\ ${ }^{b}$ Theoretical Physics Department, CERN, Geneva, Switzerland \\ ${ }^{c}$ Center for Cosmology, Particle Physics and Phenomenology (CP3), \\ Université Catholique de Louvain, 1348 Louvain-La-Neuve, Belgium \\ ${ }^{d}$ SLAC National Accelerator Laboratory, Stanford University, Stanford, CA 94309, USA \\ ${ }^{e}$ Department of Physics, University of Zürich, CH-8057 Zürich, Switzerland \\ E-mail: jbroedelephysik.hu-berlin.de, claude.duhrecern.ch, \\ dulatf@slac.stanford.edu, b.penante@cern.ch, aprimo@physik.uzh.ch \\ lorenzo.tancrediecern.ch
}

In this contribution to the proceedings of Loops and Legs in Quantum Field Theory 2018, we discuss some recent developments in the calculation of multiloop Feynman integrals which evaluate to functions beyond multiple polylogarithms.

Loops and Legs in Quantum Field Theory (LL2018)

29 April 2018 - 04 May 2018

St. Goar, Germany

${ }^{*}$ Speaker. 


\section{Introduction and motivations}

In the last years it has become clear that functions beyond multiple polylogarithms are ubiquitous in particle physics. They made their first appearance already in 1962 in the calculation of the two-loop QED corrections to the electron self-energy by A. Sabry [1], and as of today have shown up in diverse two-loop corrections in QCD and EW theory, as soon as enough scales and (in particular) massive virtual particles are considered [2-20]. It is for this reason that developing tools to handle these functions analytically and numerically will be essential for the success of the ambitious physics program to be carried out at the LHC.

In order to trace the origin of these functions in Feynman integral calculations, it is useful to start from the by now renowned method of differential equations [21-24]. The method is based on the existence of integration-by-parts identities (IBPs) among Feynman integrals [25, 26], which allow the reduction of large families of Feynman integrals to small groups of master integrals (MIs). One can then use the IBPs to derive differential equations in the kinematic invariants and the masses for the MIs, which often turn out to be easier to solve compared to a direct integration over the loop momenta of the MIs. If we call $\vec{I}(d ; \vec{x})$ the vector of the, say, $n$ distinct MIs, where $d$ is the number of space-time dimensions and $\vec{x}$ is the vector of the Mandelstam invariants, the differential equations take the schematic form

$$
\frac{\partial}{\partial x_{k}} \vec{I}(d ; \vec{x})=C_{k}(d ; \vec{x}) \vec{I}(d ; \vec{x})
$$

where the $C_{k}(d ; \vec{x})$ are $n \times n$ matrices whose coefficients are rational functions in $d$ and the $x_{j}$. Now, the components of the vector of MIs $\vec{I}(d ; \vec{x})$ should be subdivided as follows

$$
\vec{I}(d ; \vec{x})=(\vec{m}(d ; \vec{x}), \vec{s}(k ; \vec{x})),
$$

where $\vec{m}(d ; \vec{x})$ are the $N$ master integrals with the largest number of propagators and which correspond to the topology under study (for example, a double box), while $\vec{s}(d ; \vec{x})$ are the $M$ "simpler" MIs, i.e. integrals with fewer propagators, also referred to as sub-topologies. Of course $n=N+M$. The differential equations then, written for the components of the vector $\vec{m}$, look as follows

$$
\frac{\partial}{\partial x_{k}}\left(\begin{array}{c}
m_{1}(d ; \vec{x}) \\
\vdots \\
m_{N}(d ; \vec{x})
\end{array}\right)=h(d ; \vec{x})\left(\begin{array}{c}
m_{1}(d ; \vec{x}) \\
\vdots \\
m_{N}(d ; \vec{x})
\end{array}\right)+\left(\begin{array}{c}
\sum_{j=1}^{M} n h_{1 j}(d ; \vec{x}) s_{j}(d ; \vec{x}) \\
\vdots \\
\sum_{j=1}^{M} n h_{N j}(d ; \vec{x}) s_{j}(d ; \vec{x})
\end{array}\right)
$$

where $h(d ; \vec{x})$ is the homogeneous matrix while $n h_{j}(d ; \vec{x})$ are the non-homogeneous contributions. Loosely speaking, the homogeneous part of the system constitutes the main source of complexity in the solution of Eq. (1.2).

Quite schematically, the solution of the differential equations proceeds as follows. First, we expand the differential equations for $d \sim 4$, which is the physical number of dimensions we are interested in. ${ }^{1}$ In doing this, up to possible rescaling of some of the master integrals by powers of $(d-4)$ and neglecting the subtopologies for simplicity, we are left with equations in the form

\footnotetext{
${ }^{1}$ Note that, as it is well known, $d \sim 4$ is equivalent to any $d \sim 2 n$, for $n \in \mathbb{N}$.
} 


$$
\frac{\partial}{\partial x_{k}} \vec{m}(d ; \vec{x})=h^{(0)}(\vec{x}) \vec{m}(d ; \vec{x})+(d-4) h^{(1)}(\vec{x}) \vec{m}(d ; \vec{x})+\mathscr{O}\left((d-4)^{2}\right),
$$

where $h(d ; \vec{x})=h^{(0)}(\vec{x})+(d-4) h^{(1)}(\vec{x})+\mathscr{O}\left((d-4)^{2}\right)$, and the $h^{(j)}(\vec{x})$ are $N \times N d$-independent matrices. Formally, a solution of the system above requires finding an $N \times N$ matrix $G(\vec{x})$ of homogeneous solutions such that

$$
\frac{\partial}{\partial x_{k}} G(\vec{x})=h^{(0)}(\vec{x}) G(\vec{x})
$$

Then, by rotating the basis of master integrals $\vec{m}(d ; \vec{x})=G(\vec{x}) \vec{f}(d ; \vec{x})$, we find that

$$
\frac{\partial}{\partial x_{k}} \vec{f}(d ; \vec{x})=(d-4) G^{(-1)}(\vec{x}) h^{(1)}(\vec{x}) G(\vec{x}) \vec{f}(d ; \vec{x})+\mathscr{O}\left((d-4)^{2}\right)+\text { subtopologies },
$$

which, by expanding also the vector of master integrals in $(d-4)$ gives

$$
\vec{f}(d ; \vec{x})=\sum_{n=0}^{\infty}(d-4)^{n} \vec{f}^{[n]}(x),
$$

allows one to formally write the solution for the Laurent coefficients of the MIs around $d=4$ as iterated integrals, i.e. at a generic order $n$ we have

$$
\vec{f}^{[n]}(\vec{x})=\int d x G^{-1}(\vec{x}) h^{(1)}(\vec{x}) G(\vec{x}) \vec{f}^{[n-1]}(\vec{x})+\text { subtopologies } .
$$

Note that above we have assumed that all MIs are properly normalized such that their Laurent expansion in $d \sim 4$ starts at order $(d-4)^{0}$. Eq. (1.6) remains rather formal and unilluminating, at least until we can clarify two substantial points:

1. How do we get the matrix $G(\vec{x})$ in general?

2. Given the matrix $G(x)$, what are the functions defined by the iterated integrals above?

In what follows we will try to give an answer these two questions, at least in some special cases of Feynman integrals of direct practical interest for phenomenological applications and which cannot be evaluated in terms of the by now standard multiple polylogarithms (MPLs) [27-30].

\section{Homogeneous solutions and unitarity cuts}

The answer to the first question turns out to be very simple and, at least in principle, very general. Indeed, finding a complete set of solutions of a generic system of differential equations of the form of Eq. (1.4) is a non-trivial task, at least if the matrix $h^{(0)}(x)$ is not in triangular form. The crucial observation is that Eq. (1.4) is not "any" system of differential equation, but instead it is satisfied by construction by a family of Feynman integrals. So the question becomes, can we use physical information of the Feynman integrals in order to infer the homogeneous solution? The answer turns out to be yes. In particular, one can show that the so called maximal-cut of the 


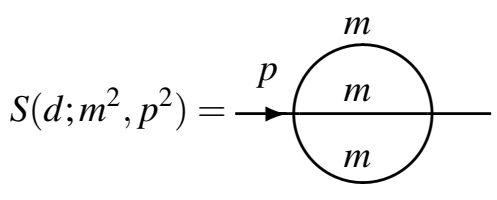

Figure 1: The two-loop sunrise graph

Feynman integrals under consideration provides the full set of homogeneous solutions required to construct the matrix $G(\vec{x})$ [31-34].

The simplest example of this very general idea was worked out for the first time in the case of the two-loop massive sunrise graph in ref. [5], see Fig. 1. We will use this case as exemplification of the idea. It is easy to show that $S\left(d ; m^{2}, p^{2}\right)$ in $d=2$ space-time dimensions satisfies the secondorder differential equation [5],

$$
\left\{\frac{d^{2}}{d z^{2}}+\left[\frac{1}{z}+\frac{1}{z-1}+\frac{1}{z-9}\right] \frac{d}{d z}-\left[\frac{1}{3 z}-\frac{1}{4(z-1)}-\frac{1}{12(z-9)}\right]\right\} S(d ; z)=R(z)
$$

where we defined $z=p^{2} / m^{2}$ and $R(z)$ is some rational function of $z$. In ref. [5], the authors realized that the imaginary part of the sunrise graph is in fact a solution of the homogeneous equation

$$
\left\{\frac{d^{2}}{d z^{2}}+\left[\frac{1}{z}+\frac{1}{z-1}+\frac{1}{z-9}\right] \frac{d}{d z}-\left[\frac{1}{3 z}-\frac{1}{4(z-1)}-\frac{1}{12(z-9)}\right]\right\} \operatorname{Im} S(d ; z)=0 .
$$

The latter can be computed by use of the Cutkosky-Veltman rules and, in this case, coincides with one possible solution for its maximal cut. A simple calculation gives (up to numerical factors)

$$
\operatorname{Im} S(d ; z) \propto \frac{1}{\sqrt{(3-\sqrt{z})(\sqrt{z}+1)^{3}}} \mathrm{~K}\left(\frac{16 \sqrt{z}}{(3-\sqrt{z})(\sqrt{z}+1)^{3}}\right),
$$

where $\mathrm{K}(x)$ is the complete elliptic integral of the first kind

$$
\mathrm{K}(x)=\int_{0}^{1} \frac{d t}{\sqrt{\left(1-t^{2}\right)\left(1-x t^{2}\right)}} .
$$

The second independent solution (which does not correspond to the discontinuity of the graph) is obtained by performing the integral which defines the maximal-cut along an independent integration contour

$$
\mathrm{Cut}_{2} S(d ; z) \propto \frac{1}{\sqrt{(3-\sqrt{z})(\sqrt{z}+1)^{3}}} \mathrm{~K}\left(1-\frac{16 \sqrt{z}}{(3-\sqrt{z})(\sqrt{z}+1)^{3}}\right),
$$

which also solves Eq. (2.2). This construction can be easily generalized to three- or four-point functions and provides a very powerful tool to write integral representations for the solutions of Feynman integrals which do not evaluate to standard polylogarithms.

The problem of what the functions defined by these iterated integrals are, remains. In the next section we will summarize some of the recent progress in this direction and define a class of functions that can be used to integrate analytically many two-loop "elliptic" Feynman integrals. 


\section{Elliptic generalizations of multiple polylogarithms}

A general consensus on what is the "correct" class of functions to express the iterated integrals generated by elliptic systems of differential equations has not been reached yet. In spite of this, a lot of progress has been made and a rather general picture is starting to emerge. Indeed, from a purely mathematical point of view, a class of functions that generalizes multiple polylogarithms to the "elliptic world" has been defined and studied by mathematicians not so long ago [35]. The mathematical construction of these functions is based on the geometrical equivalence between an elliptic curve and (the universal covering of) a complex torus, i.e. a Riemann surface of genus 1. This definition of multiple elliptic polylogarithms has found large applicability in the computation of open-string one-loop amplitudes [36-38]. Unfortunately, their direct use to the computation of multiloop Feynman integrals is not obvious. To remedy this problem, more recently an alternative formulation of these functions has been put forward, which uses instead the language of iterated integrals over elliptic curves [39-41], which appear more naturally in the direct calculation of Feynman integrals. Let us briefly recap the definition of these functions. We start with an elliptic curve defined by a quartic polynomial

$$
y^{2}=P_{4}(x)=\left(x-a_{1}\right)\left(x-a_{2}\right)\left(x-a_{3}\right)\left(x-a_{4}\right),
$$

where the $a_{j}$ will be considered constant in what follows. In ref. [39], the elliptic polylogarithms are defined as iterated integrals of the form

$$
\mathrm{E}_{4}\left(\begin{array}{ccc}
n_{1} & \ldots & n_{k} \\
c_{1} & \ldots & c_{k}
\end{array} ; t\right)=\int_{0}^{t} d x \psi_{n_{1}}\left(c_{1}, x\right) \mathrm{E}_{4}\left(\begin{array}{cccc}
n_{2} & \ldots & n_{k} \\
c_{2} & \ldots & c_{k}
\end{array} ; x\right)
$$

where the integration kernels that will be relevant in the following are ${ }^{2}$

$$
\psi_{0}(0, x)=\frac{c_{4}}{y}, \quad \psi_{1}(c, x)=\frac{1}{x-c}, \quad \psi_{-1}(c, x)=\frac{y_{c}}{y(x-c)}, \quad \psi_{-1}(\infty, x)=\frac{x}{y},
$$

with $c \in \mathbb{C}, y_{c}=\sqrt{P_{4}(c)}$ and $c_{4}=\frac{1}{2} \sqrt{\left(a_{1}-a_{3}\right)\left(a_{2}-a_{4}\right)}$. In ref. [40] it was shown that the functions $\mathrm{E}_{4}$ are in close correspondence to the multiple elliptic polylogarithms defined in [35] and that it is straightforward to go from one formulation to the other by a suitable change of variables.

The functions $E_{4}$ turn out to be very useful to express analytically different examples of elliptic Feynman integrals and also the $\varepsilon$-expansion of some non trivial classes of Hypergeometric functions. To show this in practice, we present some preliminary results which will appear soon, with more details, in a more complete publication. Let us consider the following two-loop, non-planar three-point function

$$
\begin{aligned}
T(d ; a) & =\overbrace{-p_{2}} \\
& =\int \frac{D^{d} k D^{d} l}{\left(k^{2}-m^{2}\right)\left(l^{2}-m^{2}\right)\left(\left(k-p_{1}\right)^{2}-m^{2}\right)\left(\left(l-p_{2}\right)^{2}-m^{2}\right)\left(k-l-p_{1}\right)^{2}\left(l-k-p_{2}\right)^{2}},
\end{aligned}
$$

\footnotetext{
${ }^{2}$ More details on the definition of the functions $\mathrm{E}_{4}$ and the complete set of kernels can be found in ref. [39].
} 
where we defined $a=-m^{2} / p^{2}$. We keep the discussion rather generic and do not specify explicitly the loop-integration measure $D^{d} k$. We notice that the integral is finite in $d=4$ and therefore does not need regularization. By introducing Feynman parameters we find in $d=4$

$$
T(4 ; a)=\int_{0}^{1} \prod_{i=1}^{6} d x_{i} \delta\left(1-\sum_{i=1}^{6} x_{i}\right) \frac{1}{\left[F\left(x_{1}, x_{2}, x_{3}, x_{4}, x_{5}, x_{6}, p^{2}, m^{2}\right)\right]^{2}}
$$

with

$$
\begin{aligned}
& F\left(x_{1}, x_{2}, x_{3}, x_{4}, x_{5}, x_{6}, p^{2}, m^{2}\right)=\left(p^{2}\left[\left(\left(x_{3}+x_{4}\right) x_{5}+x_{2}\left(x_{3}+x_{5}\right)\right) x_{6}+x_{1} x_{5}\left(x_{4}+x_{6}\right)\right]\right. \\
& \left.+m^{2}\left(x_{1}+x_{2}+x_{3}+x_{4}\right)\left(x_{3} x_{4}+x_{5} x_{4}+x_{6} x_{4}+x_{3} x_{5}+x_{3} x_{6}+x_{2}\left(x_{3}+x_{5}+x_{6}\right)+x_{1}\left(x_{2}+x_{4}+x_{5}+x_{6}\right)\right)\right) .
\end{aligned}
$$

By use of the Cheng-Wu theorem ${ }^{3}$, it is relatively easy to perform all integrations but one and write the three-point function above as

$$
\begin{aligned}
& T(4 ; a)=\frac{1}{3} \int_{0}^{1} d t \frac{a^{2}}{\sqrt{(t-1) t} \sqrt{4 a+(t-1) t}}\left\{6 \left[G((t-1) t, a)\left(G_{m}\left(-\frac{a}{t-1}, t\right)+2 G_{m}(t, t)\right)\right.\right. \\
& +G(0, t)\left(2 G_{m}(t, t)-G_{m}\left(\frac{a}{t-1}+t, t\right)\right)-G(1, t) G_{m}\left(\frac{a}{t-1}+t, t\right)+2 G_{m}\left(0, \frac{a}{t-1}, t\right) \\
& +G_{m}\left(-\frac{a}{t-1}, \frac{a-t^{2}+t}{1-t}, t\right)+2 G_{m}\left(t, \frac{a-t^{2}+t}{1-t}, t\right)+G_{m}\left(\frac{a}{t-1}+t, \frac{a}{t-1}, t\right) \\
& \left.-2 \log (a) G_{m}(t, t)+\log (a) G_{m}\left(\frac{a}{t-1}+t, t\right)+2 G(1, t) G_{m}(t, t)\right]-6 G_{m}(t)[G(0, t) G((1-t) t, a) \\
& \left.\left.+G(1, t) G((1-t) t, a)+G(0,(1-t) t, a)+G(0,(t-1) t, a)-\log (a) G((1-t) t, a)+\pi^{2}\right]\right\}
\end{aligned}
$$

where we defined

$$
G_{m}(\vec{n}, x)=G\left(r_{p}, \vec{n}, x\right)-G\left(r_{m}, \vec{n}, x\right), \quad r_{p / m}=\frac{-t(1-t) \pm \sqrt{(t-1) t} \sqrt{4 a+t(t-1)}}{2(t-1)},
$$

and $G(\vec{n}, x)$ are the usual multiple polylogarithms. By inspection of the formulas above we see immediately that $y^{2}=P_{4}(x)=x(x-1)(4 a+x(x-1))$ defines an elliptic curve and that each of the multiple polylogarithms $G_{m}$ can be easily expressed as combinations of elliptic polylogarithms $\mathrm{E}_{4}$. As exemplification, at length one we have

$$
\begin{gathered}
G_{m}(t)=G\left(r_{p}, t\right)-G\left(r_{m}, t\right)=\log \left(\frac{t(1-t)+\sqrt{P_{4}(t)}}{t(1-t)-\sqrt{P_{4}(t)}}\right) \\
\frac{\partial}{\partial t} G_{m}(t)=\frac{1-2 t}{\sqrt{(t-1) t} \sqrt{4 a-t(1-t)}}
\end{gathered}
$$

and by integrating back in $t$ we find

$$
G_{m}(t)=\int_{0}^{t} \frac{d x}{\sqrt{P_{4}(x)}}-2 \int_{0}^{t} \frac{d x x}{\sqrt{P_{4}(x)}}+c=\frac{4}{1+\sqrt{1-16 a}} \mathrm{E}_{4}\left({ }_{0}^{0} ; t\right)-2 \mathrm{E}_{4}\left(\begin{array}{c}
-1 \\
\infty
\end{array} ; t\right)+c
$$

\footnotetext{
${ }^{3}$ We integrate the Feynman parameters using the ordering suggested in [42].
} 
Where $c$ is an integration constant. By rewriting consistently all $G$ and $G_{m}$ in terms of $\mathrm{E}_{4}$ with argument $t$, it becomes straightforward to perform the last integration in $t$ and obtain

$$
\begin{aligned}
& T(4, a)=\frac{2 a^{2}}{c_{4}^{2}}\left[5 \mathrm{E}_{4}\left(\begin{array}{cccc}
0 & 0 & 1 & 1 \\
0 & 0 & 0 & r_{m p}
\end{array} ; 1\right)+5 \mathrm{E}_{4}\left(\begin{array}{cccc}
0 & 0 & 1 & 1 \\
0 & 0 & 0 & r_{p p}
\end{array} ; 1\right)+5 \mathrm{E}_{4}\left(\begin{array}{cccc}
0 & 0 & 1 & 1 \\
0 & 0 & 1 & r_{m p}
\end{array} ; 1\right)+5 \mathrm{E}_{4}\left(\begin{array}{cccc}
0 & 0 & 1 & 1 \\
0 & 0 & 1 & r_{p p}
\end{array} ; 1\right)\right. \\
& -3 \mathrm{E}_{4}\left(\begin{array}{cccc}
0 & 0 & 1 & 1 \\
0 & 0 & r_{m m} & 0
\end{array} ; 1\right)-3 \mathrm{E}_{4}\left(\begin{array}{cccc}
0 & 0 & 1 & 1 \\
0 & 0 & r_{m m} & 1
\end{array} ; 1\right)-3 \mathrm{E}_{4}\left(\begin{array}{cccc}
0 & 0 & 1 & 1 \\
0 & 0 & r_{p m} & 0
\end{array} ; 1\right)-3 \mathrm{E}_{4}\left(\begin{array}{cccc}
0 & 0 & 1 & 1 \\
0 & 0 & r_{p m} & 1
\end{array} ; 1\right) \\
& \left.+3 \log a\left(\mathrm{E}_{4}\left(\begin{array}{ccc}
0 & 0 & 1 \\
0 & 0 & r_{m m}
\end{array} ; 1\right)+\mathrm{E}_{4}\left(\begin{array}{ccc}
0 & 0 & 1 \\
0 & 0 & r_{p m}
\end{array} ; 1\right)\right)\right]
\end{aligned}
$$

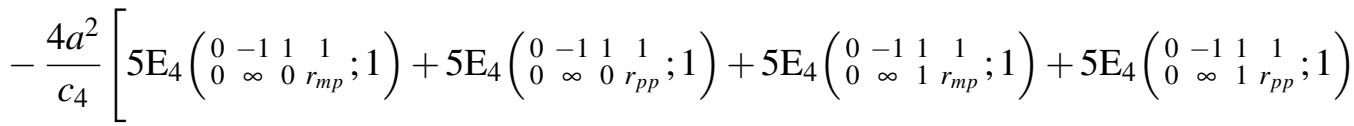

$$
\begin{aligned}
& -3 \mathrm{E}_{4}\left(\begin{array}{cccc}
0 & -1 & 1 & 1 \\
0 & \infty & r_{m m} & 0
\end{array} ; 1\right)-3 \mathrm{E}_{4}\left(\begin{array}{cccc}
0 & -1 & 1 & 1 \\
0 & \infty & r_{m m} & 1
\end{array} ; 1\right)-3 \mathrm{E}_{4}\left(\begin{array}{cccc}
0 & -1 & 1 & 1 \\
0 & \infty & r_{p m} & 0
\end{array} ; 1\right)-3 \mathrm{E}_{4}\left(\begin{array}{cccc}
0 & -1 & 1 & 1 \\
0 & \infty & r_{p m} & 1
\end{array} ; 1\right) \\
& \left.+3 \log a\left(\mathrm{E}_{4}\left(\begin{array}{ccc}
0 & -1 & 1 \\
0 & \infty & r_{m m}
\end{array} ; 1\right)+\mathrm{E}_{4}\left(\begin{array}{ccc}
0 & -1 & 1 \\
0 & \infty & r_{p m}
\end{array} ; 1\right)\right)\right]
\end{aligned}
$$

with

$$
c_{4}=\frac{1+\sqrt{1-16 a}}{4}, r_{m m / p}=\frac{1-\sqrt{1 \mp 4 a}}{2}, r_{p m / p}=\frac{1+\sqrt{1 \mp 4 a}}{2},
$$

This is the result we were seeking. Still, we notice that Eq. (3.4) is not optimal. In fact, since the singularities $r_{m / p, m / p}$ lie on the integration contour, in order to make sense of Eq. (3.4) one needs either to deform the integration contour, or to rewrite this expression in a form in which every single $\mathrm{E}_{4}$ function is explicitly well defined. Performing these optimizations is beyond the scope of these proceedings, where we instead aimed to show how the recently introduced $\mathrm{E}_{4}$ functions can be used in order to solve a realistic and physically relevant family of Feynman integrals. A more suitable representation for this three-point function in terms of well defined elliptic polylogarithms will be presented elsewhere.

\section{Conclusions}

In this short contribution we have discussed recent advancements in the calculation of multiloop Feynman integrals which evaluate to functions beyond multiple polylogarithms. First, we have shown that the differential equation method, augmented by information provided by the maximal cut of the relevant Feynman integrals, allows to write solutions for arbitrarily complicated Feynman integrals in terms of iterated integrals over rational functions and products of the maximal cut solutions. In the second half of this proceedings, we showed instead how a non-planar two-loop three-point function can be written in terms of elliptic multiple polylogarithms. This is the first example of a three-point function which can be expressed in terms of this class of functions. While a lot of work still remains to be done to merge the language of elliptic polylogarithms and that of differential equations (see for example [43]), these recent developments are very promising and open the way to a systematic study of complicated Feynman integrals relevant for precision physics calculations at the LHC. 


\section{Acknowledgements}

This research was supported by the the ERC grant 637019 "MathAm" and the U.S. Department of Energy (DOE) under contract DE-AC02-76SF00515.

\section{References}

[1] A. Sabry, Nucl. Phys. 33 (1962), no. 17 401-430.

[2] D. J. Broadhurst, Z. Phys. C47 (1990) 115-124.

[3] S. Bauberger, F. A. Berends, M. Bohm, and M. Buza, Nucl. Phys. B434 (1995) 383-407, [hep-ph/9409388].

[4] S. Bauberger and M. Bohm, Nucl. Phys. B445 (1995) 25-48, [hep-ph/ 9501201$].$

[5] S. Laporta and E. Remiddi, Nucl.Phys. B704 (2005) 349-386, [hep-ph / 0406160 ].

[6] M. Czakon and A. Mitov, Nucl. Phys. B824 (2010) 111-135, [arXiv: 0811.4119].

[7] S. Bloch and P. Vanhove, J. Number Theor. 148 (2015) 328-364, [arXiv: 1309 . 5865].

[8] L. Adams, C. Bogner, and S. Weinzierl, J.Math.Phys. 54 (2013) 052303, [arXiv: 1302.7004 ].

[9] L. Adams, C. Bogner, and S. Weinzierl, J. Math. Phys. 55 (2014), no. 10102301 , [arXiv:1405.5640].

[10] L. Adams, C. Bogner, and S. Weinzierl, J. Math. Phys. 56 (2015), no. 7072303 , [arXiv:1504.03255].

[11] L. Adams, C. Bogner, and S. Weinzierl, arXiv:1512.05630.

[12] L. Adams, C. Bogner, A. Schweitzer, and S. Weinzierl, arXiv:1607.01571.

[13] J. Ablinger, A. Behring, J. Blümlein, A. De Freitas, A. von Manteuffel, and C. Schneider, Comput. Phys. Commun. 202 (2016) 33-112, [arXiv:1509.08324].

[14] E. Remiddi and L. Tancredi, Nucl. Phys. B907 (2016) 400-444, [arXiv: 1602.01481$].$

[15] R. Bonciani, V. Del Duca, H. Frellesvig, J. M. Henn, F. Moriello, and V. A. Smirnov, JHEP 1612 (2016) 096 arXiv:1609.06685.

[16] A. von Manteuffel and L. Tancredi, JHEP 06 (2017) 127, [arXiv: 1701.05905 ].

[17] L.-B. Chen, J. Jiang, and C.-F. Qiao, JHEP 1804, 080 (2018), arXiv: 1712.03516.

[18] J. L. Bourjaily, A. J. McLeod, M. Spradlin, M. von Hippel, and M. Wilhelm, Phys. Rev. Lett. 120, no. 12, 121603 (2018) arXiv: 1712.02785.

[19] J. Ablinger, A. Behring, J. Blümlein, A. De Freitas, E. Imamoglu, M. van Hoeij, A. von Manteuffel, C. G. Raab, C. S. Radu, and C. Schneider, arXiv:1711.09742.

[20] B. Mistlberger, JHEP 1805, 028 (2018) arXiv: 1802.00833.

[21] A. Kotikov, Phys.Lett. B254 (1991) 158-164.

[22] E. Remiddi, Nuovo Cim. A110 (1997) 1435-1452, [hep-th/ 9711188 ].

[23] T. Gehrmann and E. Remiddi, Nucl.Phys. B580 (2000) 485-518, [hep-ph/ 9912329 ].

[24] J. M. Henn, Phys.Rev.Lett. 110 (2013) 251601, [arXiv: 1304 . 1806]. 
[25] F. Tkachov, Phys.Lett. B100 (1981) 65-68.

[26] K. Chetyrkin and F. Tkachov,Nucl.Phys. B192 (1981) 159-204.

[27] A. B. Goncharov, Adv. Math. 114 (1995) 197-318.

[28] E. Remiddi and J. Vermaseren, Int.J.Mod.Phys. A15 (2000) 725-754, [hep-ph/9905237].

[29] A. B. Goncharov, Math. Res. Lett. 5 (1998) 497-516, [arXiv: 1105.2076 ].

[30] J. Vollinga and S. Weinzierl, Comput.Phys.Commun. 167 (2005) 177, [hep-ph / 0410259 ].

[31] A. Primo and L. Tancredi, Nucl. Phys. B916 (2017) 94-116, [arXiv: 1610 . 08397 ].

[32] J. Bosma, M. Sogaard, and Y. Zhang, JHEP 1708, 051 (2017) arXiv: 1704.04255.

[33] A. Primo and L. Tancredi, Nucl. Phys. B921 (2017) 316-356, [arXiv: 1704.05465 ].

[34] M. Harley, F. Moriello, and R. M. Schabinger, JHEP 06 (2017) 049, [arXiv: 1705.03478 ].

[35] F. Brown and A. Levin, arXiv:1110.6917.

[36] J. Broedel, C. R. Mafra, N. Matthes, and O. Schlotterer, JHEP 07 (2015) 112, [arXiv:1412.5535].

[37] J. Broedel, N. Matthes, and O. Schlotterer, arXiv:1507.02254.

[38] J. Broedel, N. Matthes, G. Richter, and O. Schlotterer, J. Phys. A51 (2018), no. 28 285401, [arXiv:1704.03449].

[39] J. Broedel, C. Duhr, F. Dulat, and L. Tancredi, JHEP 1805, 093 (2018) arXiv: 1712.07089.

[40] J. Broedel, C. Duhr, F. Dulat, and L. Tancredi, Phys. Rev. D 97, no. 11, 116009 (2018) arXiv:1712.07095.

[41] J. Broedel, C. Duhr, F. Dulat, B. Penante, and L. Tancredi, arXiv:1803.10256.

[42] M. Hidding and F. Moriello, arXiv:1712.04441.

[43] J. Broedel, C. Duhr, F. Dulat, B. Penante and L. Tancredi, arXiv: 1807.00842. 